\title{
Restoration of Chest X-ray by Kalman Filter
}

\author{
Jinwoo Kim, Member, KIMICS
}

\begin{abstract}
A grid was sandwiched between two cascaded imaging plates. Using a fan-beam X-ray tube and a single exposure scheme, the two imaging plates, respectively, recorded grid-less and grid type information of the object. Referring to the mathematical model of the Grid-less and grid technique, it was explained that the collected components whereas that of imaging plates with grid was of high together with large scattered components whereas that of imaging plate with grid was of low and suppressed scattered components. Based on this assumption and using a Gaussian convolution kernel representing the effect of scattering, the related data of the imaging plates were simulated by computer. These observed data were then employed in the developed post-processing estimation and restoration (kalman-filter) algorithms and accordingly, the quality of the resultant image was effectively improved.
\end{abstract}

Index Terms - Kalman filter, Computed Radiography, Xray tube, scattering, $X$-ray imaging.

\section{INTRODUCTION}

AS the Computed Radiography systems using photostimulable phosphor imaging plate were developed [1] to permit digital image generation which excelled in linearity and reproducibility, many research institutes conducted researches on computer-aided image processing with a view toward image diagnostic value enhancement[2]. One of the main practical obstacles on obtaining a high-contrast X-ray image is the problem of scattering. The effect of scattered radiation is acquiring an obscured X-ray image with poor detectable lesions and hence a vague diagnostic information. A good review of anti-scatter grids, air gap methods, and slit scanning systems are well documented in [3], [4] as to their efficacy in reducing scatter degradations. However, grids and air gaps can not completely eliminate the X-ray scatter reaching the detector and slit scanning systems suffer from motion artifacts and high X-ray tube loading [5]. Under such circumstances, post-processing

\footnotetext{
Manuscript received August 3, 2010; revised August 31, 2010; accepted September 3, 2010 .

Jinwoo Kim is with the Department of Information \& Communication, Kyungsung University, Busan, 607-736, Korea (Email: jinwoo@ks.ac.kr)
}

techniques have been employed to improve the quality of the resultant X-ray image with either sampling the scatter throughout the image using radio-opaque beam-stops [6], [7], or modeling the scatter mathematically and then correcting it using digital filtering techniques [1]-[5], [7][13]. As it was explained in [5], the techniques that use radio-opaque beam-stop require additional exposure to directly sample the intensity of scattered radiation and can not be applied in the case of consecutive image acquisition such as cardiac imaging. On the other hand, the previously mentioned digital filtering techniques due to the uncertainty in describing the mathematical models of the X-ray imaging system, imperfect measuring of scattering and system parameters, the existence of patient body as a dominant source of noise, etc. can not satisfactorily cope with clinical practice and still need more improvement. Presented in this investigation is a novel data acquisition and manipulation technique in the single-exposure X-ray imaging systems: Two imaging plates in a cascaded scheme have been utilized to collect the imaging-target data. The two imaging plates, respectively, record grid-less and grid types information. The main purpose of this research is to provide a lowerdose X-ray imaging system while the acquired image quality compared with the existing $\mathrm{X}$-ray machines is preserved.

\section{THE GRID-LESS AND GRID DATA ACQUISITION TECHNIQUE}

As it is shown in Fig. 1, a grid is located between two photostimulable phosphor imaging plates. The top imaging plate 1 records the X-ray information passed through the object without any filtering, whereas the bottom imaging plate 2 records the X-ray information propagated through an anti-scatter grid. Using this technique, imaging plate 1 records a high data with large scattered components whereas imaging plate 2 records a low data with reduced scattered components. $\mathrm{X}$-ray systems that only use imaging plate with an anti-scatter grid, in contrast to those of without grid, require a higher exposure to achieve a better image contrast. In other words, a higher radiation risk is the cost of acquiring a superior result. The purpose of the Grid-less and grid data acquisition technique (Fig. 1) is to obtain an image with the same quality as of a conventional X-ray system, and hopefully a lower 
exposure requirement.

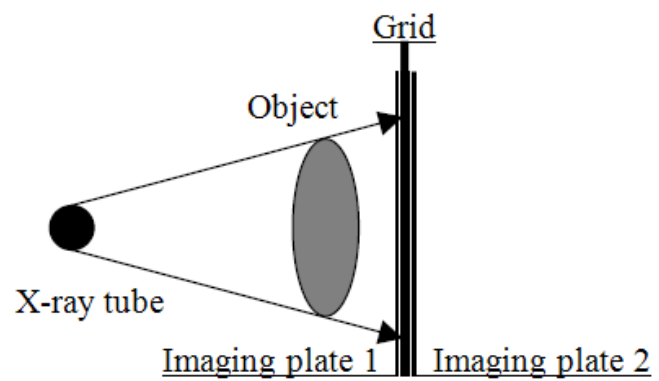

Fig. 1. Grid-less and grid data acquisition technique.

\section{METHODS}

\section{A. Mathematical Model for the Acquired images}

As X-rays propagate through an object, the transmitted photons incident on an imaging plate either do not interact or are scattered one more times. These primary and scattered components thus form a mutually exclusive additive set [4]. Similar to the previous works [3]-[5], [7], [13], and with considering the effect of noise the detected intensity of the imaging plates on a pixel basis can be described as

$$
d_{k}(x, y)=p_{k}(x, y)+s_{k}(x, y)+n_{k}(x, y)
$$

where, $d_{k}(x, y), p_{k}(x, y)$, and $s_{k}(x, y)$, refer to detected, primary and scattered photon components that reach to pixel $(x, y)$ of imaging plate $k(k=1,2)$; and $n_{k}(x, y)$ represent the corresponding noise. The scattered radiation distribution $\left[s_{k}(x, y)\right]$ can be approximated by $2 \mathrm{D}$ convolution of scatter point spread function $\left[g_{k}(x, y)\right]$ with $p_{k}(x, y)$ [7]-[9], [5]

$$
s_{k}(x, y)=R_{k} p_{k}(x, y) * g_{k}(x, y)
$$

where $R_{k}$ represents the scatter intensity as a fraction of primary intensity $\left[p_{k}(x, y)\right]$, the symbol $(*)$ demotes $2 \mathrm{D}$ convolution operation, and $g_{k}(x, y)=\exp \left[-\left(x^{2}+y^{2}\right) / 2 \sigma_{k}^{2}\right]$. Using (2), (1) can be written as follows

$$
d_{k}(x, y)=p_{k}(x, y) * h_{k}(x, y)+n_{k}(x, y)
$$

where $k=1,2$, and

$$
h_{k}(x, y)=\delta_{k}(x, y)+R_{k} g_{k}(x, y)
$$

In the same reasons as of the previous works [5], [7][9], we have used a Gaussian function to represent the convolution kernel, i.e. $g_{k}(x, y)$ also, the effect of the grid on the primary intensity has been considered as only linear attenuation, i.e. if $p 1=p$ then $p 2=\beta p$ where $\beta<1$. Moreover, the effects of displacement, enlargement, and depositioning of the grid that may cause any registration problem between the components of imaging plates have been presently neglected. Referring to the above statements, (3) can be expressed as follows

$$
\begin{aligned}
& d_{1}(x, y)=p(x, y) * h_{1}(x, y)+n_{1}(x, y) \\
& d_{2}(x, y)=p(x, y) * \beta h_{2}(x, y)+n_{2}(x, y)
\end{aligned}
$$

In this research, our goal was to estimate $p_{k}(x, y)$, using 1) the recorded data of the imaging plates $\left(d_{k}(x, y) ; k=1\right.$, 2), 2) given Gaussian convolution kernel with unknown parameters, and 3) known stochastic behavior of noise. Referring to the fact that in the actual X-ray images the unknown parameters of $\sigma_{1}, \sigma_{2}, R_{1}$, and $R_{2}$ are spatially variable, the above equations may remain valid if and only if we employ them for dealing with a small area of $\mathrm{X}$-ray images. This may validate the assumption of the constancy for the parameters.

\section{B. Estimation of Scatter Parameters}

In general, the intensity of scattered radiation depends on the exposure parameters (beam energy, field size, air gap, projection angle) and object thickness. Due to the complexity of imaging targets in some parts of the body such as the chest, finding the perfect scatter parameters on a pixel by pixel basis can be far from a simple matter. For this reason, several assumptions such as 1) considering scatter with locally constant parameters (see the usage of beam-stop array in [7]), 2) having some information about the anatomical structure of the object (see mammographic imaging in [13]), 3) using a previously made look-uptable of the X-ray data [5], [11], etc. were made to simplify the problem. In this work, we have assumed that in a small area of the X-ray image with a size of $M X M$ $(\mathrm{M}<<\mathrm{N})$, the scatter point-spread function i.e. $g_{k}(x, y)$, can be expressed by a Gaussian function as follows

$$
g_{k}(x, y)=\exp \left[-\left(x^{2}+y^{2}\right) / 2 \delta_{k}^{2}\right]
$$

where $k=1,2$. Accordingly, using (7) and taking 2D FFT of (5) and (6) results

$$
\begin{aligned}
& D_{1}(u, v)=P(u, v)\left[1+R_{1} G_{1}(u, v)\right]+N_{1}(u, v) \\
& D_{2}(u, v)=P(u, v) \beta\left[1+R_{2} G_{2}(u, v)\right]+N_{2}(u, v)
\end{aligned}
$$

where the capital letters show the FFT counterparts and

$$
G_{k}(u, v)=2 \pi \sigma_{k}^{2} \exp \left[-\left(u^{2}+v^{2}\right) / 2\left(1 / \delta_{k}^{2}\right)\right]
$$

Assuming that the components of signal compared with those of noise are superior in the low frequency regions of 
the Fourier domain, (8) and (9) can be approximated by

$$
\begin{gathered}
D_{1}(u, v) \approx P(u, v)\left[1+R_{1} G_{1}(u, v)\right] \\
D_{2}(u, v) \approx P(u, v) \beta\left[1+R_{2} G_{2}(u, v)\right]
\end{gathered}
$$

and hence

$$
\frac{D_{1}(u, v)}{D_{2}(u, v)} \approx \frac{1+R_{1} G_{1}(u, v)}{\beta\left[1+R_{2} G_{2}(u, v)\right]}
$$

Finally, substitution of (10) in (13) results

$$
\frac{D_{1}(u, v)}{D_{2}(u, v)} \approx \frac{1+2 \pi R_{1} \sigma_{1}^{2} \exp \left[-\left(u^{2}+v^{2}\right) / 2\left(1 / \sigma_{1}^{2}\right)\right]}{\beta\left[1+2 \pi R_{2} \sigma_{2}^{2} \exp \left[-\left(u^{2}+v^{2}\right) / 2\left(1 / \sigma_{2}^{2}\right)\right]\right]}
$$

As it was reported in [16], the plain X-ray films of images of high resolution can be digitized by $4 \mathrm{~K} \times 4 \mathrm{~K} \times 12$ bit digitizers, i.e. $N \approx 4000$. Hence, by using (14) in a small area of the X-ray image $(M<<N ; M=64$, for example), we can obtain $M X M$ equations (4096, for $M=64)$ for dealing with only five unknown parameters $\left(R_{l}\right.$, $R_{2}, \sigma_{1}, \sigma_{2}$ and $\beta$ ). We solved the above equations by applying the Levenberg-Marquardt nonlinear least square algorithm [16]. In practice and in order to obtain good results, particularly due to the presence of noise in the high-frequency regions of the Fourier domain, we only used the central $M / 2$ points of the horizontal $(u)$ axes. Accordingly, the number of employed equations were decreased to $(M / 2) \times(M / 2)$. Also, referring to the effect of grid, it was assumed that $\sigma_{1}>>\sigma_{2}, R_{1}>>R_{2}$, and $\beta<1$. These assumptions were made to obtain good initial values for the Levenberg-Marquardt nonlinear search algorithm [15]. The above discussed method is applicable to the various areas of the imaging plates and demonstrates the possible capability of the Grid-less and grid system to estimate X-ray image scatterrelated parameters with spatially-varying nature.

\section{The Kalman Filter Equations}

A Kalman filter algorithm is a statistical approach to estimate a time varying state vector from noisy measurement based on a model of linear vector differential or difference equation [14]. In this work, the Kalman filter is employed to match the model of the Grid-less and grid X-ray system, the statistics of the error between the model and observation, and the uncertainty associated with the measurements. Using a size $N X N$ for imaging plates. In the following equations, capital letters denote matrix operations while lowercase characters represent vectors.

System Dynamic:

$$
p(j+1 \mid j)=\phi p(j)+u(j)
$$

Measurement Equation:

$$
d(j)=H(j) p(j)+n(j)
$$

where $j$ is the image column $(j=1, \cdots, N) ; \phi$ is a constant; $p^{T}(j)=[p(j, 1) \cdots p(j, N)]$; the error of the model from reality is given by a $N x 1$ vector, $u(j)$, a zeromean Gaussian white noise of variance $\sigma_{u}^{2}$ and covariance matrix $R_{u} ; d(j)=\left[d_{l}(j) d_{2}(j)\right]$ is a $1 \times 2 N$ vector; $d_{1}(j)$ and $d_{2}(j)$, respectively represent the pixels corresponding to columns $j$ of imaging plates, i.e.

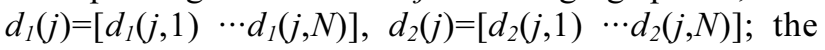
observation matrix $H(j)$ is size of $2 N X N$.

$$
H(j)=\left[\begin{array}{l}
H_{1}(j) \\
H_{2}(j)
\end{array}\right]
$$

where $H_{1}(j)$ and $H_{2}(j)$ are size of $N_{X} N$, and respectively denote the convolution kernels corresponding to imaging plate 1 and imaging plate 2, the measurement noise is given by a $1 \times 2 N$ vector, $n(j)=\left[n_{l}(j) \quad n_{2}(j)\right]$ where $n_{l}(j)$ and $n_{2}(j)$ are $1 \times N$ vectors denoting zero-mean Gaussian white noise of variance $\sigma^{2}{ }_{n 1}$ and the $\sigma^{2}{ }_{n 2}$ and covariance matrix of $R_{n 1}$ and $R_{n 2}$ corresponding to imaging plate 1 and imaging plate 2 , respectively, the covariance matrix of $n(j)$ is size of $2 N \times 2 N$ represented by $R_{n}$. Based on the above model, the Kalman-filtering operation can be viewed as consisting of the following steps

State Prediction:

$$
\hat{p}(j \mid j-1)=\phi \hat{p}(j-1 \mid j-1)
$$

Kalman Gain:

$$
K(j)=E(j \mid j-1) H^{T}(j)\left[H(j) E(j \mid j-1) H^{T}(j)+R_{n}\right]^{-1}
$$

State Correction:

$$
\hat{p}(j \mid j)=\phi \hat{p}(j-1 \mid j-1)+K(j)[d(j)-H(j) \hat{p}(j \mid j-1)]
$$

Convariance Correction:

$$
E(j \mid j)=[1-k(j) H(j)] E(j \mid j-1)
$$

Covariance Prediction:

$$
E(j+1 \mid j)=\phi^{2} E(j \mid j)+R_{u}
$$

\section{EXPERIMENTAL RESULTS}

In this section, the discussed methods of the previous Sections have been evaluated with the computer simulations. In the experiments, we have used the following Gaussian kernel to represent the blur-function 


$$
b_{k}(j, i)=\exp \left[-i^{2} / 2 \sigma_{k}^{2}(j)\right]
$$

where $k=1,2$. In $(23), b_{k}(j, i)$ demonstrates a onedimensional blur along the Y-axis with spatially variable variance $\left(\sigma^{2}{ }_{\mathrm{k}}(j)\right)$ along the $\mathrm{X}$-axis. Hence, in the simulations, $g_{k}(x, y)$ was replaced with $b_{k}(x, y)$. Referring to (4), it can be concluded that with employing (23) as the blur-function, we should estimate four unknown parameters at each column (the Y-axis) of the image. In this work, we have applied first-order auto-regressive AR models to simulate these parameters as follows

$$
\begin{gathered}
\sigma_{k}(j+1)=\psi_{k} \sigma_{k}(j)+v_{k}(j) \\
R_{k}(j+1)=\rho_{k} R_{k}(j)+t_{k}(j)
\end{gathered}
$$

where $k=1,2 ; \psi_{k}$ and $\rho_{k}$ are constants; $v_{k}(j)$ and $t_{k}(j)$ are estimation error of the AR models and can be represented by Gaussian white noises.

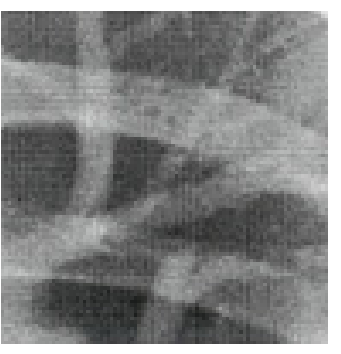

(a) Original image

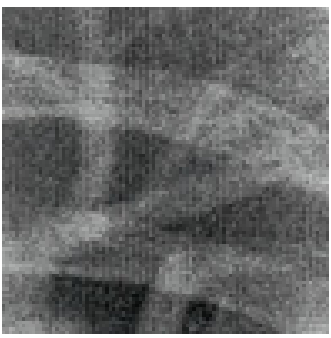

(c) imaging plate 2

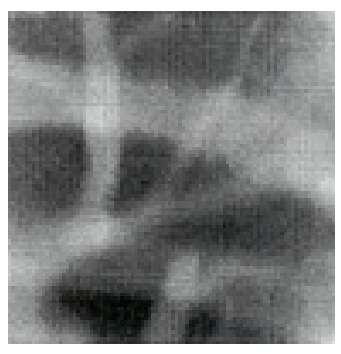

(b) imaging plate 1

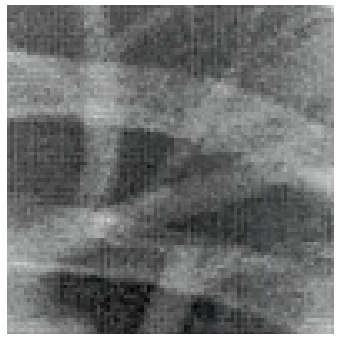

(d) Grid-less and grid
Fig. 2. Evaluation of the Grid-less and grid X-ray imaging technique with the chest $\mathrm{X}$-ray data.

Fig. 2(a) shows the original image (size: 256x256) employed in simulation studies. Fig. 2(b) and (c), respectively are the generated imaging plate 1 and imaging plate 2 images by applying (5), (6), and (23)(25). As long as the associated $(\mathrm{S} / \mathrm{N})$ of the imaging plate 1 and imaging plate 2 were respectively larger the 40 and $10 \mathrm{dBs}$, by using the method explained in Section III-B, we succeeded to find all unknown parameters $\left(\beta ; \sigma_{l}(j), \sigma_{2}(j), R_{l}(j)\right.$, and $R_{2}(j) ; j=1,2, \cdots$, $N)$ with less than $5 \%$ errors. The estimated parameters were then used in the Kalman filter algorithm to restore the original image using the observed images of imaging plate 1 and imaging plate 2 . The result is shown in Fig. 2(d). It was confirmed that the Grid-less and grid imaging technique was effective to improve the quality of noisy-blurred images with spatially-varying unknown scatter-parameters. In a SUN (ULTRA 2) system, the reconstruction time for $N=256$ was approximately 40 minutes. Extension of the ideas of estimation and reconstruction of the 1D spatially variable scatter to the 2D case by replacing (15) and (23) with their corresponding 2D models is also applicable. However, the dimension of the state for Kalman filter may be very large for $2 \mathrm{D}$ solutions. It means that in this case, the optimal kalman filter with extensive computation should be replaced with a suboptimal but efficient alternative.

\section{COMCLUSION}

We have proposed the Grid-less and grid system as a new data acquisition alternative to the aim of providing a high quality low dose X-ray imaging system. Following the successful estimation of spatially varying scatter parameters in a computer simulation study, a Kalman filter was employed to effectively restore the image information. Although the results of the simulation study were encouraging, we need further investigations as well as more practical assumptions to experience the behavior of the techniques in the situation. Such attempts are presently under development.

\section{ACKNOWLEDGMENT}

This research was supported by Kyungsung University Research Grants in 2010.

\section{REFERENCES}

[1] M. Sonoda, M. Takano, J. Miyahara, and H. kato, "Computed radiography utilizing scanning laser stimulated luminescence," Radiology, vol.148, no.3, pp. 833-838, Sep. 1983.

[2] W. Ito, K.Shimura, N. Nakajima, M. Ishida, and H. Kato, "Improvement of detection in computed radiography by new single-exposure dual-energy subtraction," J Digit Imaging, vol.6, no.1, pp. 42-47, Feb. 1993.

[3] S. W. Smith, and R. A. Kruger, "A signal processing model of diagnostic x-ray scatter," Med. Phys., vol.13, no.6, pp. 831-835, Nov-Dec. 1986.

[4] J. A. Seibert, and J. M. Boone, "X-ray scatter removal by deconvolution," Med. Phys., vol.15, no.4, pp. 567-575, Jul-Aug. 1988.

[5] A. Ersahin, S. Molloi, and Y. J. Qian, "A digital filtration technique for scatter-glare correction based on thickness estimation," IEEE Trans. Med. Img., vol.14, no.3, pp. 587-595, Sep. 1995.

[6] C. G. Shaw, and D. B. Plewes, "Quantitative digital subtraction angiography: two scanning techniques for correction of scattered 
radiation and veiling glare," Radiology, vol.157, no.1, pp. 247-253, Oct. 1985.

[7] L. A. Love, and R. A. Kruger, "Scatter estimation for a digital radiographic system using convolution filtering," Med. Phys., vol.14, no.2, pp. 178-185, Mar-Apr. 1987.

[8] C. G. Shaw, D. L. Ergun, P. D. Myerowitz, M. S. Van Lysel, C. A. Mistretta, W. C. Zarnstorff, and A. B. Crummy, "A Technique of scatter and glare correction for video densitometric studies in digital subtraction video angiography," Radiology, vol.142, pp. 209-213, Jan. 1982.

[9] R. A. Kruger, and P. Y. Liu, "Digital angiography using a matched filter," IEEE Trans. Med. Img., vol.1, no.1, pp. 16-21, 1982.

[10] T. N. Hangartner, "Correction of scatter in computed tomography images of bone," Med. Phys., vol.14, no.3, pp. 335-340, May-Jun. 1987.

[11] S. Y. Molloi, and C. A. Mistretta, "Scatter-glare correction in quantitative dual-energy fluoroscopy," Med. Phys., vol.5, pp. 289297, 1988.

[12] H. Fahighnam, and A. Macovski, "Reducing the effects of scattered photons in x-ray projection imaging," IEEE Trans. Med. Img., vol.8, no.1, pp.56-63, 1989.

[13] R. P. Highnam, J. M. Brady, and B. J. Shepstone, "Computing the scatter component of mammographic images," IEEE Trans. Med. Img., vol.13, no.2, pp.301-313, 1994.

[14] R. E. Kalman, "A new approach to linear filtering and prediction problems," ASME Trans.J. Basic Eng., vol.D, pp.35-45, Mar. 1960.

[15] J. E. Dennis, and R. B. Schnabel, "Numberical Methods for Unconstrained Optimization and Nonlinear Equations," INew Jersy: Prentice-Hall, 1983.

[16] S. Wong, 1. Zaremba, D. Gooden, and H. K. Huang, "Radiologic image compression - a review," Proc. IEEE, vol.83, pp.194-219, Feb. 1995.

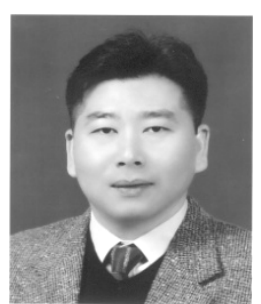

Jinwoo Kim received the B.S degree in Electrical Engineering from Myongji University in 1992 and the M.S and Ph.D. degrees in Electronic Engineering and System design Engineering from Fukui University, Fukui, Japan, in 1996 and 1999, respectively. From 2000 to 2003, he was a contract Professor in the Department of Information Communication and Computer Engineering at Hanbat National University, Daejeon, Korea. Since 2003 he has been with the Department of Information and Communication Engineering at Kyungsung University, Busan, Korea, where he is currently an associate professor. From Dec., 2007 to Mar., 2009, he was a visiting researcher in the Department of Bioengineering at Tokyo University, Japan. His current research interests include image processing, pattern recognition, and medical imaging technology. 